\title{
A COMMON LENS NUCLEAR AREA IN SCHEIMPFLUG PHOTOGRAPHS
}

\author{
WEN QIAN, PER G. SÖDERBERG, ENPING CHEN, KARIN MAGNIUS, BO PHILIPSON \\ Stockholm, Sweden
}

\begin{abstract}
SUMMARY
Scheimpflug photography has been proven to be a useful method for documentation of opacification of the lens nucleus. In the present study, a common lens nuclear area (CNA) is established for photometry of nuclear cataract on Scheimpflug photographs. Using the established method, one measurement for one photograph, and one photograph for each subject is enough for the photometry of nuclear density. Further, this method allows detection of a 10\% change in nuclear density between two occasions with a group of at least 7 subjects, and for independent groups with at least 95 subjects in each group $(\alpha=0.05$, $\beta=0.10$ ). It is anticipated that the CNA will provide a useful tool for quantitative determination of nuclear cataract.
\end{abstract}

In epidemiological studies on cataract and for evaluation of the efficiency of anti-cataract drugs, a standard system for quantitative recording of lens opacities is needed. Scheimpflug photography is a method for objective recording of lens opacification. Cortical opacities manifest considerable rotational asymmetry in density. ${ }^{1,2}$ However, human nuclear cataracts show considerable symmetry around the anterior-posterior pole, and one Scheimpflug photographic section is believed to represent the nuclear density with high precision. ${ }^{1,3}$ Therefore, Scheimpflug photography has been used for quantitative documentation of lens nuclear cataract. $^{2-5}$ Various methods for measuring lens nuclear opacity on Scheimpflug photographs have been proposed. ${ }^{3-8}$

Densitometry of Scheimpflug photographs was introduced by Hockwin et al. ${ }^{4,5}$ The photograph was scanned along the optical axis of the lens, and the amplitude of the density and the integral of the density within an interval along the axis were estimated. ${ }^{4,5}$ However, a single densitometric scan samples only a small fraction of the nuclear region of the image and the result may be affected by the choice of line position by the analyst. Multilinear scans

From: Research Laboratory, St. Erik's Eye Hospital, and Department of Medical Biophysics, Karolinska Institute, Stockholm, Sweden.

Correspondence to: Wen Qian, Research Laboratory, St. Erik's Eye Hospital, S-112 82 Stockholm, Sweden. generate large amounts of data and therefore require considerable computer capacity. ${ }^{3}$

Mayer et al. ${ }^{6}$ used digital image subtraction for evaluation of changes in a Scheimpflug image. With this method, two digitised Scheimpflug photographs taken on different occasions are superimposed and then subtracted. Pixels with the same brightness are cancelled out, while pixels with different brightness remain visible. This technique assesses the changes in the density of the whole lens. The procedure requires sophisticated equipment.

Chylack et $a .^{3}$ defined two different nuclear masks for analysis of digitised Scheimpflug photographs. One was an outer shell which encloses all of the nucleus within the zones of enhanced supranuclear scatter, and the other was an inner shell which encloses only the fetal-embryonal zones of the nucleus. The mean density of the mask was considered as the nuclear density.

Kashiwagi and Khu introduced ${ }^{7}$ and evaluated ${ }^{8}$ a colour component subtraction method for measuring nuclear opacification using colour Scheimpflug photographs. In this method the colour photograph was illuminated with white light. Densitometries for red, green and blue colour of the image were performed simultaneously along the anterior-posterior axis of the lens within the nuclear area. Then, one curve was subtracted from another providing three different curves: red minus green, green minus blue and blue minus red. For each difference curve, the difference in colour as a function of position was fitted to a firstorder polynomial with linear regression. The regression coefficient estimated was considered a response variable for cataract.

It is reasonable to expect variability in location and size of the nuclear area among lenses. Moreover, the border between the cortex and nucleus is usually indistinguishable on Scheimpflug photographs. These factors make it difficult to settle a general rule for the selection of a nuclear area for density measurements. A common lens nuclear area (CNA), which does not contain the cortex, could facilitate evaluation of nuclear opacity for epidemiological studies of nuclear cataract.

In order to record any change of density in the nuclear 


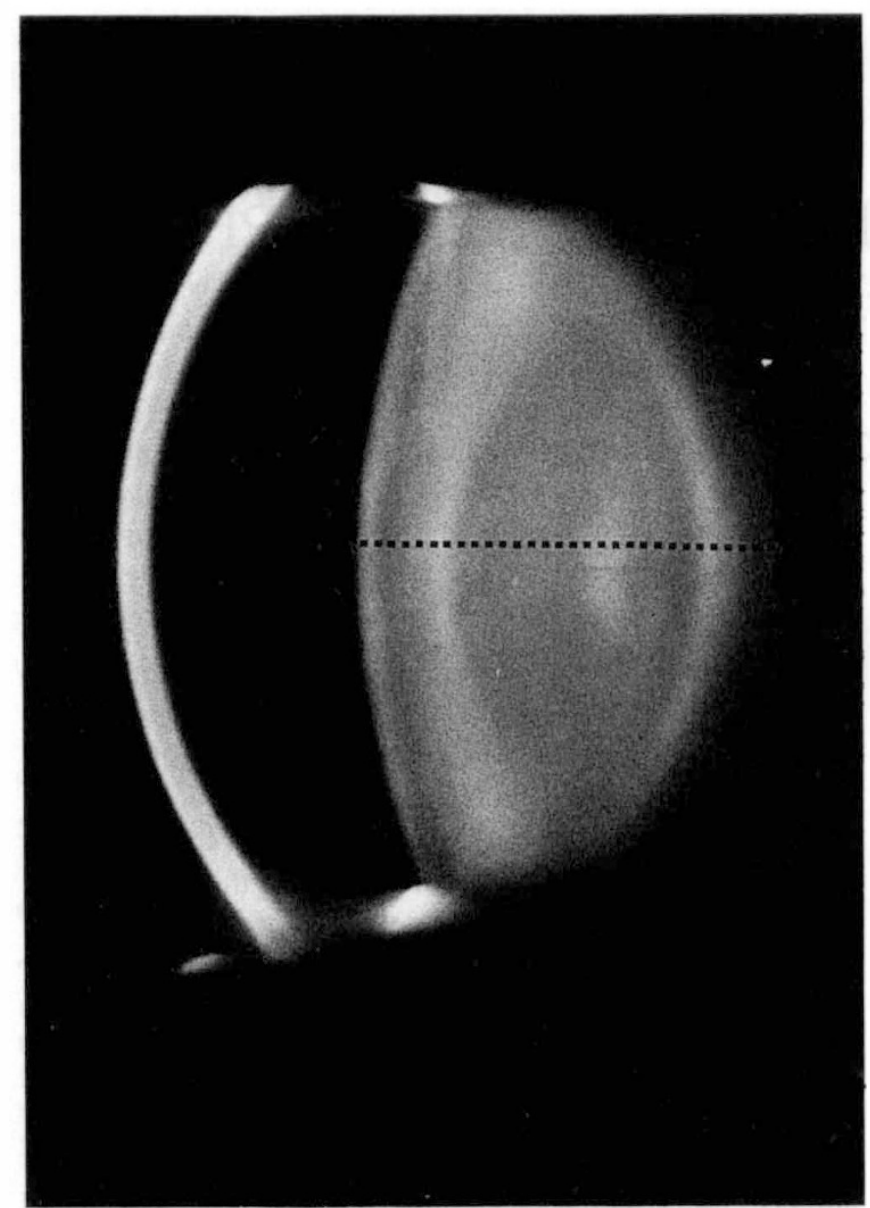

(a)

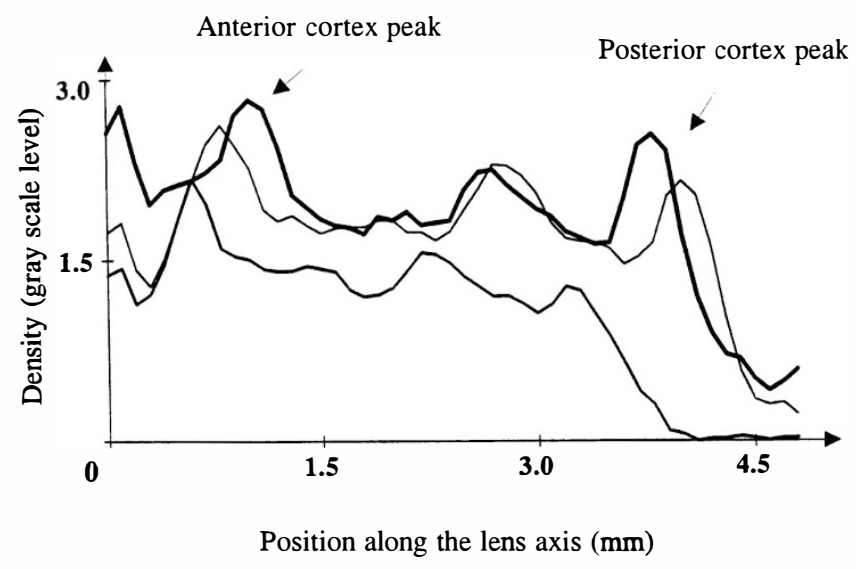

(b)

area, the CNA should be as large as possible. However, the cortex must be excluded in order to obtain high precision. The purpose of the present study was to determine a CNA that fulfils these requirements of photometry of nuclear cataract in Scheimpflug photographs.

\section{MATERIALS AND METHODS}

\section{Calibration of Dimension on a Scheimpflug Photograph}

A microscopic metric scale was photographed with the Scheimpflug camera. The magnification of the size of the lens on a Scheimpflug photograph was determined by
Fig. 1. (a) Scheimpflug image. The dashed line corresponds to stepwise scanning along the anatomical lens axis with a photometer. (b) Unfiltered scans of density along the anatomical lens axis in three different lenses. (c) Filtered scans of density. Filled circles, the point of transition between anterior cortex and nucleus; open circles, the point of transition between posterior cortex and nucleus.

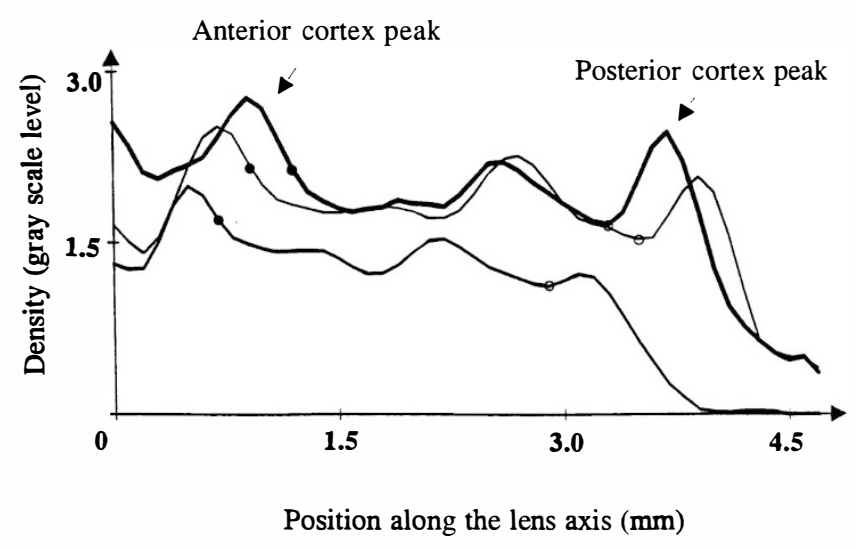

(c)

comparing the image of the scale with the original. This allowed calibration of the Scheimpflug image to metric scale.

\section{Determination of a Common Lens Nuclear Area (CNA)}

Lens photographs were taken with a Topcon SL-45 slit lamp camera with a built-in reference system consisting of a five-step grey scale. Kodak TX 5063 film was used. All films were developed according to a standard procedure. ${ }^{9}$ Ten Scheimpflug photographs, each from a different person (aged 52-70 years, 8 males and 2 females), were 
selected randomly from a continuing clinical project. All selected lenses were clear at slit lamp examination.

To determine a point of transition between anterior cortex and nucleus, and between posterior cortex and nucleus, respectively, the lens image was scanned stepwise along the anatomical lens axis with a photometer (Fig. 1a). The photometer consisted of a photomultiplier incorporated into a Leitz microscope.$^{10}$ The Scheimpflug photograph was put on a holder which was integrated into the microscope and combined with an $X-Y$ coordinate system. The $X-Y$ movement of the holder could be recorded. The magnification during measurement was 125 times. The anatomical lens axis was determined as the normal to the pupillary plane intersecting the centre of the pupil. The measuring spot of the photometer was $0.10 \times 0.10 \mathrm{~mm}$. The interval between two spots was $0.01 \mathrm{~mm}$. A photometric reading was converted to corresponding grey scale level by a third-order polynomial, which was derived from the grey scale reference in each photograph. ${ }^{11}$ Three examples of the density variation along the lens axis are shown in Fig. $1 b$.

The density along the lens axis was digitally filtered according to equation (1) to reduce the variability within a short distance along the axis (Fig. 1c):

$$
F\left(x_{i}\right)=\left(x_{i-1}+x_{i}+x_{i}+{ }_{1}\right) / 3
$$

Here, $F\left(x_{i}\right)$ is a filtered density at the $(i)$ th point. $x_{i-1}, x_{J}$ and $x_{i+1}$ were the original densities at the $(i-1)$ th, $(i)$ th and $(i+1)$ th point, respectively.

On the filtered densitometric curve, the first inflexion point after the anterior cortical peak was defined as the point of transition between anterior cortex and nucleus (Fig. 1c). The first minimum in front of the posterior cortex peak was defined as the point of transition between posterior cortex and nucleus (Fig. 1c).

A tolerance limit for the position of the transition point in the population was estimated considering the transition points in the measured lenses. For this purpose, it was assumed that the positions of the transition points in different subjects belong to a normal distribution. The risk of wrongly including cortex in the CNA was set at $2.5 \%$ for anterior and posterior cortex, respectively. Tolerance limits were estimated with tables for small samples. ${ }^{12}$

To settle the anterior and posterior curvature, respectively, for the CNA, the shapes of both the anterior and the posterior cortex were estimated in the photographs. For these measurements, the $X$-axis was a tangent to the anterior vertex of the lens and the $Y$-axis coincided with the anatomical lens axis (Fig. 2). Each lens was scanned 13 times parallel to the $Y$-axis with an inter-scan distance of $0.5 \mathrm{~mm}$ (Fig. 2). The coordinate for the density peak corresponding to the anterior and posterior cortex, respectively, was recorded.

These coordinates were separately fitted to different parabolas (equation 2) for the anterior and posterior cortex, respectively, with regression.

$$
Y=a+b X^{2}
$$

Adopting this model, the curvature of the cortex is determined by parameter $b$.

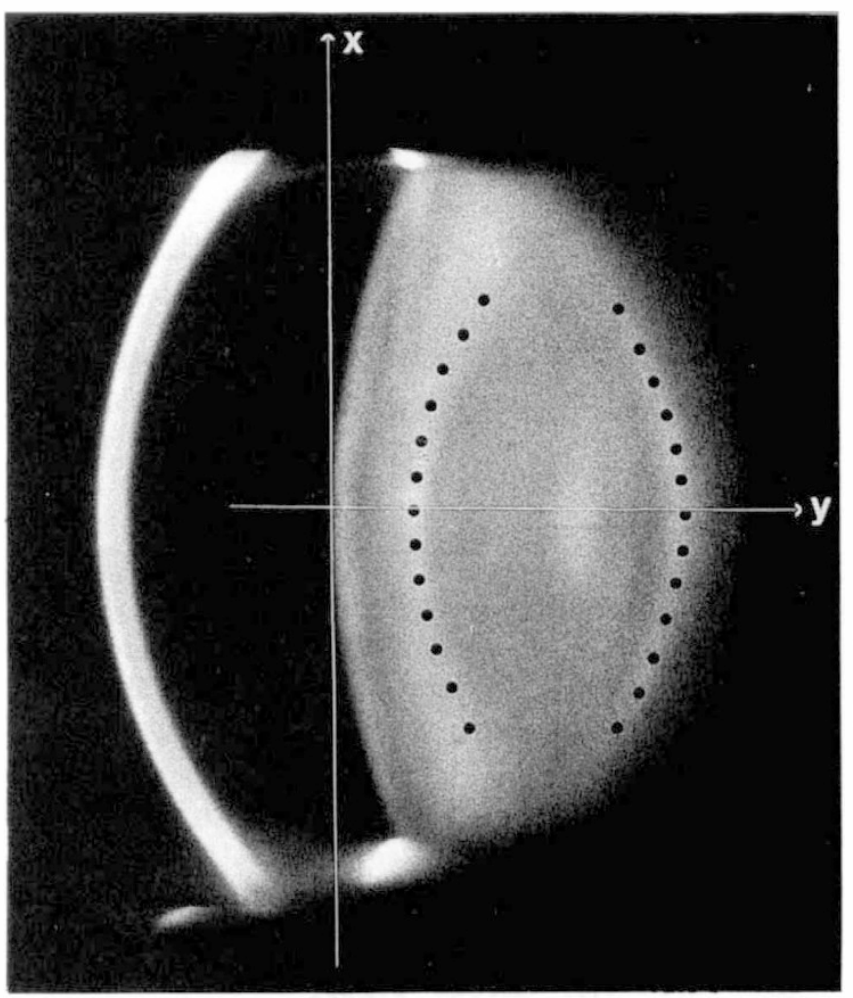

Fig. 2. Scheimpflug image. Filled circles, sample points for measurement at the anterior and posterior cortex.

For simplicity it was assumed that the values of parameter $b$ for subjects belonging to the same population are normally distributed. On this assumption a tolerance interval was estimated for $b$ in the population adopting the tolerance tables for small samples. ${ }^{12}$ The highest absolute value in the tolerance intervals for $b$ was selected for determination of the CNA.

\section{Analysis of Precision in Determination of Density in the CNA}

\section{Analysis of Sources of Variation in Photometry of Nuclear Density}

Ten rolls of film, each from a different subject, were selected randomly from a continuing clinical project. All subjects had non-cataractous lenses as judged by visual acuity and clinical examination with a slit lamp microscope. From each roll, two photographs were chosen at random. Each photograph was measured twice. The photometer used in this study limited the measuring spot to a square of $0.2 \times 0.2 \mathrm{~mm}$. For a more extended area the illumination spot was not homogeneous. Considering the spot size used and to facilitate the measurement, a rectangle within the CNA, which contains 22 of those spots, was selected for photometry of nuclear density. The mean density for the spots was considered the density of the rectangle. A photometric reading was converted to corresponding grey scale level. ${ }^{11}$ The sources of variation for the photometry of nuclear density were analysed with an analysis of variance (Appendix 1).

\section{Analysis of the Variation Among Determinations of CNA Densities}

Independent-Group-Comparison Design. The variance 


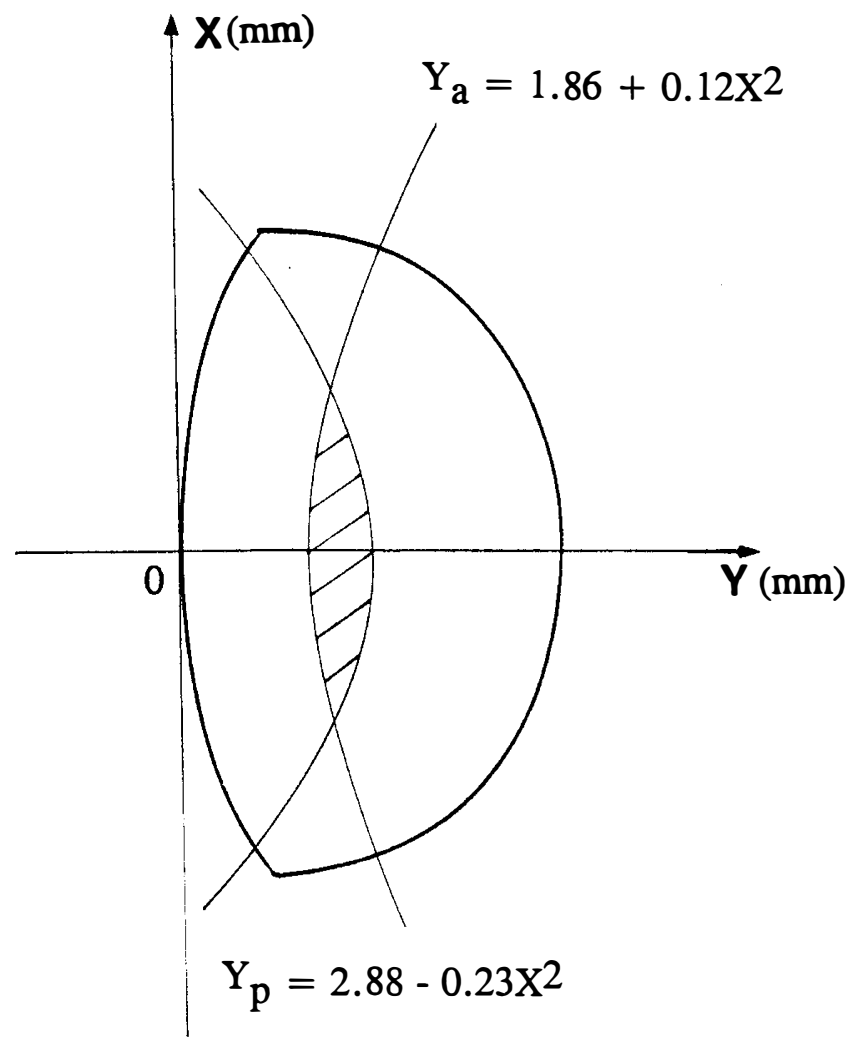

Fig. 3. Schematic drawing of a lens image. The area enclosed by the parabolas (shadowed area) was defined as the CNA.

for self-controlled design between two groups was estimated on the basis of photometry of ten pairs of lens images taken at two occasions in a group of subjects with non-cataractous lenses as judged by visual acuity and clinical examination with a slit lamp microscope. The variance components in the measurements were estimated with an analysis of variance (Appendix 2).

Paired-Comparison Design. The variance for paired design was estimated by considering the difference between two occasions for one subject as one observation.

\section{Selection of Statistical Parameters}

The significance level $(\alpha)$ and the $\beta$ level was set to 0.05 and 0.10 , respectively, considering the small sample size.

\section{RESULTS}

The image of the scale taken by the Scheimpflug camera was found to be $1: 1$ to the original.

The point at which there is a $2.5 \%$ risk of including the anterior cortex in the nuclear region is positioned

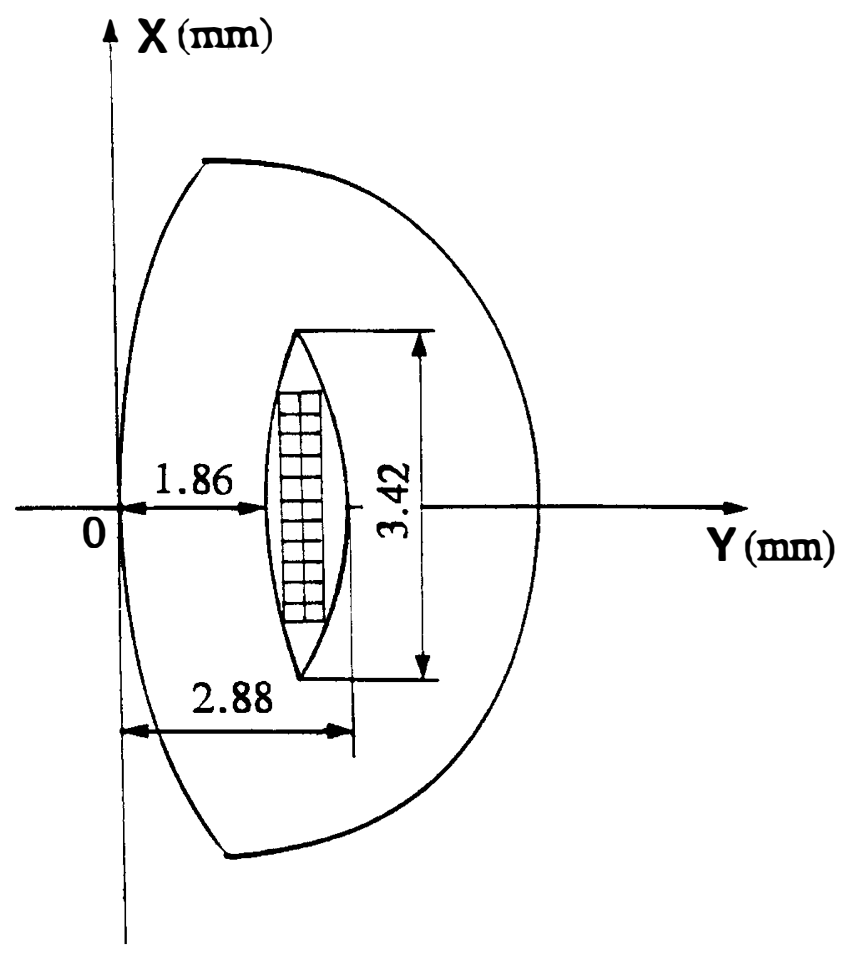

Fig. 4. Schematic drawing of a lens image. The rectangle within the CNA containing 22 small squares was selected for photometry.

$1.86 \mathrm{~mm}$ from the anterior vertex of the lens. The point at which there is a $2.5 \%$ risk of including the posterior cortex in the nuclear region is positioned $2.88 \mathrm{~mm}$ from the anterior vertex of the lens (Fig. 3).

The parameter $b$ determining the curvature of the parabola ( $b$ in equation 2 ) limiting the CNA anteriorly was 0.12 . The parameter $b$ determining the curvature of the parabola limiting the CNA posteriorly was -0.23 (Fig. 3).

The CNA is thus defined as the area enclosed between the anterior parabola $\left(Y_{\mathrm{a}}=1.86+0.12 X^{2}\right)$ and the posterior parabola $\left(Y_{\mathrm{p}}=2.88-0.23 X^{2}\right)$ (Fig. 3).

The result of the analysis of variance for photometry of nuclear density is given in Table I. The components of variance were estimated from the mean squares according to the expected mean squares (Table I). The variance for measurements $\left(\sigma_{\varepsilon}^{2}\right)$ and photographs $\left(\sigma_{\mathrm{B}}^{2}\right)$, respectively, was $0.03 \times 10^{-2}$ and $0.20 \times 10^{-2}$ (grey scale steps) ${ }^{2}$. The variance for subjects $\left(\sigma_{A}^{2}\right)$ was $10.38 \times 10^{-2}$ (grey scale steps) $)^{2}$.

The analysis of variance for determination of CNA densities is given in Table II. The variance components were

Table I. Analysis of sources of variation for photometry of nuclear density

\begin{tabular}{lcrr}
\hline $\begin{array}{l}\text { Source of } \\
\text { variation }\end{array}$ & $\begin{array}{c}\text { Degrees of } \\
\text { freedom }\end{array}$ & $\begin{array}{c}\text { Sum of squares } \\
(\text { grey scale steps) }\end{array}$ & $\begin{array}{c}\text { Mean square } \\
(\text { grey scale steps) }\end{array}$ \\
\hline Subjects & 9 & $37756 \times 10^{-4}$ & $\begin{array}{c}\text { Expected mean } \\
\text { square }\end{array}$ \\
Photographs & 10 & $430 \times 10^{-4}$ & $4195 \times 10^{-4}$ \\
Measurement & 20 & $65 \times 10^{-4}$ & $\sigma_{\varepsilon}^{2}+n \sigma_{B}^{2}+b n \sigma_{A}^{2}$ \\
\hline
\end{tabular}

$b$, number of photographs within the same film $(=2) ; n$, number of measurements on the same photograph $(=2)$. 
Table II. Analysis of variance for determination of CNA densities

\begin{tabular}{lccc}
\hline $\begin{array}{l}\text { Source of } \\
\text { variation }\end{array}$ & $\begin{array}{c}\text { Degrees of } \\
\text { freedom }\end{array}$ & $\begin{array}{c}\text { Sum of squares } \\
\text { (grey scale steps) }\end{array}$ & $\begin{array}{c}\text { Mean square } \\
\text { (grey scale steps) }^{2}\end{array}$ \\
\hline Subjects & 9 & $15081 \times 10^{-4}$ & $\begin{array}{c}\text { Expected mean } \\
\text { square }\end{array}$ \\
Occasions & 10 & $1719 \times 10^{-4}$ & $\sigma_{\varepsilon}^{2}+n \sigma_{A}^{2}$ \\
\hline
\end{tabular}

$n$, number of occasions for the same subject $(=2)$.

estimated from the mean squares according to the expected mean squares (Table II). The variance for subjects $\left(\sigma_{\mathrm{A}}^{2}\right)$ and occasions $\left(\sigma_{\varepsilon}^{2}\right)$, respectively, was $7.52 \times 10^{-2}$ and $1.72 \times 10^{-2}$ (grey scale steps) ${ }^{2}$.

The variance for paired design was estimated to be $1.48 \times 10^{-2}$ (grey scale steps). ${ }^{2}$

The minimal sample size for different experimental designs has been summarised in Table III.

\section{DISCUSSION}

In the present study, a CNA was defined for determination of the nuclear density of a lens in a Scheimpflug photograph.

Most methods for measurement of lens nuclear cataract are based on linear densitometry. Then, only a narrow line in the nuclear zone along the lens axis is measured on the photograph. But an increased density may be located outside the optical axis, especially in early-stage nuclear cataract. Thus, to optimise the possibility of detecting a density, the largest possible area in the nucleus should be measured on the photograph. However, the cortex must be excluded in order to obtain high precision. ${ }^{1,2}$ The CNA meets these requirements. Other techniques for measurement covering a nuclear area have been described. ${ }^{3}$ In these techniques the size of the area measured is determined individually. The CNA is universal for all lens images, which simplifies the measurement procedure and allows comparison of measurements from different research centres.

There is a variation of the film emulsion in Scheimpflug photographs. ${ }^{11}$ It was found that this variability is negligible with a spot size $\geqslant 0.01 \mathrm{~mm}^{2}$. ${ }^{11}$ Therefore, the spot size for image scanning was selected to be $0.1 \times 0.1 \mathrm{~mm}$. For the measurement of the nuclear region, a square $0.2 \times 0.2 \mathrm{~mm}$ was selected as the measuring spot (Fig. 4). The spot size was limited due to restriction in the photometer.

In the present study the risk of wrongly including the anterior cortex is $2.5 \%$. At the same time, the risk of wrongly including posterior cortex is $2.5 \%$. Therefore, the established CNA has a low risk of including the cortex and thus should provide a reliable sample of the nuclear density.

The analysis of variance for sources of variation for photometry of nuclear density (Table I) revealed that the variability among photographs and measurements, respectively, is negligible in relation to the variability among individuals. Consequently, one measurement in one photograph for each subject is enough for estimation of an average level of grey scale density in the CNA in a population.

The analysis of variance for determination of CNA densities (Table II) showed that the variance for occasions far exceeds that for photographs and measurements (Table I). Thus, for the self-controlled design also it is sufficient to take one measurement in one photograph for each subject.

It is appreciated that in a future study the variability in response to an experimental factor will add to the interindividual variability. This will reduce the precision. Therefore, the current estimates of sample size (Table III) should be considered as absolute minima.

The striking difference, as regards the minimum number of subjects required, between the independentgroups-comparison design and the paired-comparison design reflects the variability of the baseline nuclear density among subjects in the population. This is a problem which can not be overcome by any further improvement of the measurement methodology. For detection of a small difference in density level between two independent groups the only solution is using a large sample size. The present finding also indicates that there is a rather large range of backscatter in the lens nucleus without any significance for visual acuity, since all the examinees had normal vision. In a future evaluation of the method it would probably be preferable to include a stray light test, ${ }^{13}$ since visual acuity may not reveal a representative measure of visual comfort.

The purpose of the present study was to localise a nuclear area that can be used universally in measurements of nuclear cataract. We determined a common lens nuclear

Table III. Estimation of the minimal sample size in experiments measuring density of the common nuclear area in the crystalline lens

\begin{tabular}{|c|c|c|c|c|c|}
\hline Type of experimental design & $\hat{\sigma}_{A}^{2}(\mathrm{Gs})^{2}$ & $\hat{\sigma}_{B}^{2}(\mathrm{Gs})^{2}$ & $\hat{\sigma}_{\varepsilon}^{2}(\mathrm{Gs})^{2}$ & $\frac{s}{\hat{\mu}}(\%)$ & $N$ \\
\hline Comparison between independent groups & 0.1038 & 0.0020 & 0.0003 & $21.2^{\mathrm{a}}$ & 190 \\
\hline $\begin{array}{l}\text { Comparison, of differences between paired } \\
\text { observations, between independent groups }\end{array}$ & 0.0172 & & & 8.52 & 32 \\
\hline Paired comparison & 0.0148 & & & 7.90 & 7 \\
\hline
\end{tabular}

$\hat{\sigma}^{2}$, estimated variance for indexed source $(A=$ subjects, $B=$ photographs and $\varepsilon=$ measurements $) ; s$, estimated standard deviation for subjects; Gs, grey scale steps; $N$, least number of subjects needed (when comparing two groups, each group needs $N / 2$ ) to demonstrate an experimentally induced alteration of $10 \%$ from baseline as statistically significant $(\alpha=0.05, \beta=0.10)$.

${ }^{\mathrm{a}} s_{\overline{\mathrm{x}}_{\mathrm{i}}}=\sqrt{ }\left(\hat{\sigma}_{\mathrm{A}}^{2}+\hat{\sigma}_{\mathrm{B}}^{2}+\hat{\sigma}_{\varepsilon}^{2}\right)$. 
area for the age group 50-70 years. The size of the CNA may be influenced by sex and age; the effect of these factors remains to be investigated. It is anticipated that the established CNA will improve the accuracy for determination of lens nuclear density. This will facilitate the comparison of nuclear density between experimental and control groups in studies of the effects of drugs for the prevention of nuclear cataract. Further, the improved accuracy should reduce the numbers of subjects needed in epidemiological studies on risk factors for cataract.

\section{APPENDIXES}

Appendix 1. Model for Analysis of Variance for Photometry of Nuclear Density ${ }^{14}$

$$
X_{i j k}=\mu+A_{i}+B_{j(i)}+\varepsilon_{k(i j)}
$$

A measurement of density, $X_{i j k}$, equals the expected total mean, $\mu$, a factor for the random variation among subjects $A_{i}(i=1, \ldots, 10)$, a factor for the random variation among photographs $B_{j(i)}(j=1, \ldots, 2)$, and a factor for the random variation among measurements $\varepsilon_{k(i j)}(k=1, \ldots, 2)$.

Appendix 2. Model for Analysis of Variation Among Determinations of CNA Densities ${ }^{14}$

$$
X_{i j}=\mu+A_{i}+\varepsilon_{j(i)}
$$

A measurement of density, $X_{i j}$, equals the expected total mean, $\mu$, a factor for the random variation among subjects $A_{i}(i=1, \ldots, 10)$, and a factor for the random variation among occasions $\varepsilon_{j(i)}(j=1, \ldots, 2)$.

We are grateful to Mr Lennart Wallerman for help with the experimental instruments. This study was supported by the Wenner-Gren Center Foundation for Scientific Research, Karolinska Institutets forskningsfonder, Kronprinsessan Margaretas Arbetsnämd För Synskadade, Swedish Society of Medicine, Gun och Bertil Stohnes Stiftelse, Synfrämjandet forskningsfonder, Carmen och Bertil Regners Fond för forskning inom Området Ögonsjukdomar, and the Medical Research Council (project no. 4204).
Key words: Common lens nuclear area (CNA), Nuclear cataract, Photometry, Scheimpflug photography.

\section{REFERENCES}

1. Chen SY, Chylack LT Jr, White O. Topcon SL-45 photography, a suitable technique for documenting nuclear but not cortical cataractous change in vivo. Invest Ophthalmol Vis Sci (ARVO Suppl) 1985;26:119.

2. Datiles MB, Edwards PA, Trus BL, Green SB. In vivo studies on cataracts using the Scheimpflug slit lamp camera. Invest Ophthalmol Vis Sci 1987;28:1707-10.

3. Chylack LT Jr, McCarthy D, Khu P. Use of Topcon SL-45 Scheimpflug slit photography to measure longitudinal growth of nuclear cataracts in vivo. Lens Res 1988;5:83-93.

4. Hockwin O, Lerman S, Ohrloff C. Investigations on lens transparency and its disturbances by microdensitometric analyses of Scheimpflug photographs. Curr Eye Res $1984 ; 3: 15-22$.

5. Hockwin O, Laser H, Kapper K. Image analysis of Scheimpflug negatives. Ophthalmic Res 1988;20:99-105.

6. Mayer H, Irion KM. New approach to area image analysis of Scheimpflug photos of the anterior eye segment. Ophthalmic Res 1985;17:106-10.

7. Kashiwagi T, Khu PM. New method of measuring nuclear cataract in color Scheimpflug photographs. Ophthalmic Res 1990;22(Suppl.1):24-8.

8. Khu PM, Kashiwagi T. Quantitating nuclear opacification in color Scheimpflug photographs. Invest Ophthalmol Vis Sci 1993;34:130-6.

9. Hockwin O, Dragomirescu V, Laser H. Measurements of lens transparency or its disturbances by densitometric image analysis of Scheimpflug photographs. Graefes Arch Clin Exp Ophthalmol 1982;219:255-62.

10. Lindström B, Philipson B. Microdensitometer system for microradiography. Histochemie 1969;17:187-93.

11. Qian W, Söderberg PG, Chen E, Magnius K, Philipson B. Evaluation of grey scale steps on Scheimpflug photographs. Acta Ophthalmol 1993;71:79-84.

12. Beyer WH. Tolerance factors for normal distributions. In: CRC handbook of tables for probability and statistics. Cleveland, Ohio: CRC, 1966:125-9.

13. Neumann AC, McCarthy GR, Locke J, Cobb B, DeLand MSN. Glare disability devices for cataractous eyes: a consumer's guide. J Cataract Refract Surg 1988;14:212-6.

14. Snedecor GW, Cochran WG. The random effects model. In: Statistical methods. 7th ed. Ames, Iowa: Iowa State University Press, 1980:238-54. 